\title{
El papel del tutor en la enseñanza de la educación a distancia: una revisión sistemática sobre el enfoque de competencias
}

\section{The tutor's role in teaching distance education: a systematic review on the competencies approach}

\author{
Flavia Massuga \\ Universidade Estadual do Centro-Oeste (UNICENTRO). Irati-PR, Brasil. \\ flavia.massuga@sistemafiep.org.br \\ Simone Soares \\ Universidade Estadual do Centro-Oeste (UNICENTRO). Irati-PR, Brasil. \\ simone.soares@sistemafiep.org.br \\ Sérgio Luis Dias Doliveira \\ Universidade Estadual do Centro-Oeste (UNICENTRO). Irati-PR, Brasil. \\ sdoliveira@unicentro.br
}

\begin{abstract}
Resumen
Considerando las particularidades y la importancia del papel del tutor en la Educación a Distancia, este estudio tuvo como objetivo identificar las competencias intrínsecas a las funciones de tutoría en esta modalidad de enseñanza. Para ello, se realizó una revisión sistemática utilizando la metodología Methodi Ordinatio, en las bases de datos del Portal de Revistas Capes; Scientific Electronic Library Online, ScienceDirect y Scopus, que cubre estudios centrados en el tema en los últimos 10 años. En total, 12 estudios cumplieron los criterios establecidos y fueron evaluados. Los resultados muestran competencias técnicas, gerenciales y sociales asociadas con el papel del tutor. Entre ellos, se destacan las competencias de proporcionar atención individualizada a los estudiantes, de utilizar los sistemas y conocimientos asociados con la tecnología de la información, de proporcionar un entorno en línea acogedor, amigable, confiable y estimulante, de demostrar empatía, de estimular la interacción, de motivar a los estudiantes y proporcionar realimentación. Las dificultades aún son evidentes en relación con las habilidades técnicas y sociales, lo que lleva a la necesidad de una dirección dirigida a su desarrollo y mejora, para que puedan ayudar en el desempeño práctico eficaz y eficiente del tutor.
\end{abstract}

Palabras clave: Educación a distancia, aprendizaje en línea, tutoría, competencia professional.

\begin{abstract}
Considering the particularities and the importance of the tutor's role in the Distance Education, this study aimed to identify the competencies intrinsic to the tutoring functions in this teaching modality. For this, a systematic review was carried out using the Methodi Ordinatio methodology, in the databases of Portal de Periódicos Capes; Scientific Electronic Library Online, ScienceDirect and Scopus, covering studies focused on the theme in the last 10 years. In total, 12 studies met the established criteria and were evaluated. The results show technical, managerial and social competencies associated with the role of the tutor. Among them, we highlight the competencies of: providing individualized attention to students, using the systems and knowledge associated with information technology, providing a welcoming, friendly, trusting and stimulating online environment, demonstrating empathy, stimulating interaction, motivating students and providing feedback. Difficulties are still evident in relation to technical and social competencies, which
\end{abstract}


leads to the need for a direction aimed at their development and improvement, so that they can assist in the effective and efficient practical performance of the tutor.

Keywords: Distance education, electronic learning, tutoring, skills.

\section{Introducción}

Actualmente, con la evolución y el desarrollo de tecnologías y metodologías dirigidas a la democratización de la enseñanza, la educación a distancia (EAD) se ha consolidado cada día como una modalidad educativa en todo el mundo (Silva, Campos, Melo \& Rodrigues, 2014). Según Qayyum y Zawacki-Richter (2019), la tendencia general es el crecimiento continuo de las inscripciones en educación a distancia, esencialmente con respecto a la educación superior en los países emergentes. En Brasil, por ejemplo, hubo un aumento del 63,8\% de 2003 a 2009, alcanzando una tasa de crecimiento anual promedio de 9,9\% entre 2009 y 2014.

Teniendo en cuenta los datos del último censo de la educación superior, se observa que la modalidad de educación a distancia en Brasil ha impulsado el aumento en el número de estudiantes que ingresan a la educación superior. En el período de 2017 a 2018, se registró una variación positiva del 27,9\% en el número de inscripciones en cursos de educación a distancia, con una participación de casi el $40 \%$ en la inscripción total con 1.373.321 nuevas entradas (Instituto Nacional de Estudios e Investigaciones Educativas [INEP], 2019).

Este aumento es el resultado, entre otros factores, del crecimiento global de la educación superior, con el aprendizaje a distancia, principalmente en modo en línea, visto como una forma de satisfacer rápidamente las demandas que requieren menos infraestructura física y costos relacionados (Qayyum \& Zawacki-Richter, 2019). Además, características como una mayor flexibilidad y la eliminación de la barrera espacio/tiempo, hacen que la educación a distancia sea una opción más favorable para muchos estudiantes (Marins \& Silva, 2015).

En este contexto, la tutoría adquiere un papel fundamental. El tutor en la modalidad de educación a distancia es responsable por hacer un seguimiento del alumno, desarrollando acciones que están relacionadas con proporcionar reflexión y motivación para superar los desafíos. Participa activamente en la práctica pedagógica, contribuyendo a los procesos de enseñanza y aprendizaje de los estudiantes.

Dadas las particularidades de la modalidad de aprendizaje a distancia, el tutor tiene numerosas responsabilidades y roles relacionados con su desempeño. Para Tenório, Teles y Tenório (2016), el tutor debe realizar el proceso de enseñanza-aprendizaje, actuando como asesor y mediador. Campbell, Gallen, Jones y Walshe (2019), destacan el papel del tutor para apoyar el autoaprendizaje, o sea, desarrollar un comportamiento de iniciativa y compromiso con las actividades de aprendizaje propuestas, proporcionando comentarios sobre las tareas y facilitando la interacción en las discusiones presentadas. Para Metz y Bezuidenhout (2018), los tutores son el primer y principal contacto del estudiante con la universidad, por lo que es un factor crítico de éxito en la aceptación del aprendizaje electrónico por parte de los estudiantes.

En vista de estos desafíos, es esencial que los tutores tengan, además de experiencia en el tema, un conjunto de competencias destinadas a ejercer sus actividades independientemente de la disciplina en la que trabajen (Adnan, 2018; Hrastinski,

El papel del tutor en la enseñanza de la educación a distancia: una revisión sistemática sobre el enfoque de habilidades. Massuga, Soares y Doliveira.

Página $\mathbf{2}$ de $\mathbf{2 6}$ 
Cleveland-Innes \& Stenbom, 2018; Metz \& Bezuidenhout 2018). El término competencia se asocia con una habilidad requerida de un individuo para realizar una determinada función y se compone básicamente de conocimientos, habilidades y actitudes (Skorková, 2016; Sousa \& Barbosa, 2018). Según Grzybowka y Lupicka (2017), las competencias se pueden subdividir en técnicas, gerenciales y sociales, cubriendo requerimientos en las diversas acciones en el ejercicio de la profesión. En ese sentido, considerando la función de tutoría, Tenório et al. (2016) afirman que las competencias ayudan a los tutores a desempeñar su papel de guía y facilitador del proceso de enseñanza-aprendizaje.

A pesar de reconocer la importancia de las competencias para el desempeño de las actividades de tutoría, aún existen dudas sobre este tema, lo que permite plantear las siguientes interrogantes: ¿Qué competencias técnicas, gerenciales y sociales son fundamentales para ejercer el papel de tutor en la educación a distancia, permeado por varias particularidades? Entre ellas, ¿cuáles se destacan? ¿Qué lagunas/dificultades aún están ressaltadas en relación con las competencias para ejercer el papel de tutor en la educación a distancia?

Para dar respuesta a estas preguntas, se llevó a cabo una revisión sistemática utilizando la metodología Methodi Ordinatio, cubriendo estudios enfocados en la temática en los últimos 10 años. Se espera que los resultados aquí presentados puedan ayudar en el desempeño de los tutores universitarios en cuanto al desarrollo de competencias clave, fundamentales para la calidad en su desempeño práctico en la educación a distancia.

\section{Educación a distancia: conceptos y características}

Aunque parezca reciente, la educación a distancia como forma de enseñanza ya era evidente a fines del siglo XIX, en 1850, cuando en Europa se registró que los agricultores y ganaderos aprendían técnicas agroforestales por correspondencia. En Brasil, el movimiento comienza aproximadamente en 1904, cuando las escuelas privadas internacionales comenzaron a ofrecer cursos pagos a través del mismo proceso de envío de materiales por correo (Marins \& Silva, 2015).

Considerando un aspecto más regulatorio, la educación a distancia en Brasil fue creada y desarrollada con base en iniciativas privadas y decretos gubernamentales, dado el crecimiento tecnológico en el país. Según Gomes (2013), en 1996 se sancionó la Ley n 9.394 de las Bases Nacionales de Educación, que comenzó a fomentar el desarrollo y la creación de programas de educación a distancia en todos los niveles y modalidades de enseñanza. Sin embargo, la citada Ley solo fue reglamentada en 2005, a partir del Decreto $\mathrm{n}^{\mathrm{o}}$ 5.622, ahora derogado y sustituido por el Decreto $\mathrm{n}^{\circ} 9057$ de 2017, que permite la provisión de educación básica y superior en la modalidad a distancia, asegurando condiciones de accesibilidad en espacios y medios utilizados (Brasil, 2017).

Conceptualmente, la educación a distancia se define en el Artículo 1 del Decreto n $9057 / 2017$ como:

la modalidad educativa en la cual la mediación didáctico-pedagógica en los procesos de enseñanza y aprendizaje ocurre con el uso de medios y tecnologías de información y comunicación, con personal calificado, con políticas de acceso, con monitoreo y evaluación compatibles, entre otros, y desarrollar

El papel del tutor en la enseñanza de la educación a distancia: una revisión sistemática sobre el enfoque de habilidades. Massuga, Soares y Doliveira.

Página 3 de 26 
actividades actividades de estudiantes y profesionales de la educación que se encuentran en diferentes lugares y horarios (Brasil, 2017).

Por lo tanto, la educación a distancia se refiere a varias formas de práctica educativa que son diferentes de las prácticas presenciales, y puede incluir medios como el correo, la radio y la televisión, hoy en día, reemplazados en gran medida por Internet que abarca la educación en línea (Gomes, 2013; Lee, 2017). Según Lee (2017), la educación en línea se considera hoy como una forma innovadora de aprendizaje a distancia, como un medio interactivo y colaborativo de interacción entre estudiantes y docentes.

El aprendizaje virtual ha mostrado un rápido crecimiento, utilizándose en varios contextos, tales como: empresas, escuelas y universidades (Kopp, Matteucci \& Tomasetto, 2012). Este avance se justifica considerando que la modalidad incrementa la accesibilidad a la enseñanza, especialmente en el nivel superior (Lee, 2017; Qayyum \& Zawacki-Richter, 2019). Por lo tanto, los estudiantes que presentan demandas tales como falta de tiempo, rutinas de trabajo turbulentas y dificultades para viajar a cursos presenciales, tienden a elegir el aprendizaje a distancia.

En comparación con la educación presencial, existen diferencias notables atribuidas a los cursos de educación a distancia, especialmente con respecto a su mayor flexibilidad. Según Marins y Silva (2015), en el aprendizaje a distancia, se prescinde de la proximidad física entre estudiantes y docentes, se produciendo una comunicación bidireccional con varios sujetos: docentes, alumnos, tutores y personal administrativo, a través de canales que facilitan el contacto, como Internet. Además, se elimina la barrera espacio/tiempo y, en la modalidad de distancia, el estudiante tiene la libertad de elegir en qué momento y lugar puede realizar sus estudios. Sin embargo, dada la mayor flexibilidad, los enfoques pedagógicos tienden a exigir una mayor participación y compromiso de los estudiantes en las prácticas de aprendizaje, teniendo que gestionar sus propios conocimientos (Setlhako, 2014; Lee, 2017).

En general, la educación a distancia se ha consolidado como una modalidad de enseñanza cada vez más buscada por los estudiantes, dadas sus características particulares que denotan una mayor flexibilidad. La modalidad difiere de la educación presencial considerando las nociones de tiempo y espacio y la forma de comunicación utilizada entre los individuos involucrados.

\section{Tutoría en educación a distancia}

En el contexto de la educación a distancia, el tutor es una figura que desempeña un papel fundamental, brindando apoyo y actuando como intermediario en el proceso de enseñanza-aprendizaje. Según Kopp et al. (2012), debido a la enseñanza remota en la educación a distancia, la interacción entre estudiantes y tutores es esencial para el desarrollo de actividades necesarias para adquirir conocimiento.

El tutor puede definirse como un assessor y mediador del aprendizaje del alumno, favoreciendo la construcción de su autonomía. Además, es visto como un vínculo de interacción que contribuye con su formación, experiencias y subjetividades en la construcción del conocimiento (Bezerra \& Carvalho, 2011). Para Martins (2003), el tutor es un intermediario que garantiza la interrelación personalizada y continua del alumno en

El papel del tutor en la enseñanza de la educación a distancia: una revisión sistemática sobre el enfoque de habilidades. Massuga, Soares y Doliveira.

Página 4 de 26 
el sistema y articula los elementos del proceso y el logro de los objetivos propuestos. A su vez, Lee, Hong y Choi (2016), en resumen, entienden que el papel principal del tutor es construir una relación dialógica con los estudiantes.

Todavía para Silva et al. (2014), el tutor es un educador que establece el diálogo, sugiere, instiga y motiva la continuidad y conclusión de los cursos. Los mismos autores denotan el cambio en el perfil del tutor, enfatizando su papel como mediador al afirmar que:

En la actualidad, existe un cambio de actitudes en las interacciones entre tutores y estudiantes, por lo que el concepto de tutoría trasciende el papel del docente como transmisor de información y conocimiento, es decir, el poseedor del conocimiento cuyos estudiantes son simples receptores, dando espacio para un rol más integral es el de ser organizador, facilitador y asesor de la construcción del conocimiento del alumno, estimulador del autoaprendizaje, por lo que actúa como socio y facilitador del alumno y las interacciones entre ellos generan trabajo conjunto y resultados satisfactorios (Silva et al. , 2014, p. 3).

Teniendo en cuenta los deberes del tutor, Lee et al. (2016) entienden que el tutor tiene 3 funciones principales. La primera es la función académica, que implica responsabilidades pedagógicas e intelectuales y está estrechamente relacionada con el papel de dirigir y proporcionar reflexiones para la construcción del pensamiento crítico. La segunda asignación se entiende como gerencial, involucrando asistencia administrativa y organizacional para el estudiante. También implica resolver dudas sobre la programación de actividades y tareas y reglas de procedimiento. Finalmente, se destaca la función técnica, que incluye el apoyo a los estudiantes para problemas técnicos relacionados con los sistemas utilizados.

Del mismo modo, Borges, Coelho Junior, Faiad y Rocha (2014), entienden que el tutor tiene funciones técnicas relacionadas con la elaboración de contenido, resolución de dudas y monitoramento del progreso académico, y funciones de motivación, relacionadas con el fomento del aprendizaje y la continuidad del curso. Para Tenório et al. (2016), las funciones se resumen en asesor y mediador, siendo que el tutor también debe actuar en el proceso de evaluación y seguimiento pedagógico, brindando indicaciones materiales, elaboración de horarios, organización de eventos, actividades extraescolares, entre otros.

Es importante tener en cuenta que la comunicación entre los estudiantes y el tutor utilizando los recursos de medios disponibles puede ocurrir en tiempo síncrono y asíncrono (Bezerra \& Carvalho, 2011). En la primera modalidad, la comunicación y la interacción son instantáneas, como los ejemplos de chat y videoconferencia. En el segundo caso, la comunicación y la respuesta no ocurren al mismo tiempo, lo que ofrece a los estudiantes la oportunidad de desarrollar sus estudios de acuerdo con su disponibilidad. En este caso, la respuesta a los foros de discusión y otras actividades de evaluación pueden citarse como ejemplos.

Teniendo en cuenta las descripciones presentadas, está claro que el tutor en línea tiene un papel más complejo que los profesores tradicionales (Metz \& Bezuidenhout, 2018). El tutor de educación a distancia realiza numerosas funciones relacionadas tanto con aspectos pedagógicos, motivacionales y orientados a la resolución de problemas relacionados con las plataformas utilizadas. Por lo tanto, es esencial para el papel del tutor, además del conocimiento específico, el desarrollo de un conjunto de competencias

El papel del tutor en la enseñanza de la educación a distancia: una revisión sistemática sobre el enfoque de habilidades. Massuga, Soares y Doliveira.

Página 5 de 26 
diferenciadas que ayuden a su desempeño práctico y, en consecuencia, contribuyan a la calidad de la educación ofrecida.

\section{Enfoque de competencia}

El término competencia apareció por primera vez en el idioma francés en el siglo $\mathrm{XV}$, y su significado se dirigió a la legitimidad y autoridad de las instituciones para tratar ciertos problemas. Un poco más tarde, en el siglo XVIII, su comprensión se extiende al nivel individual, relacionándose con la capacidad y el conocimiento (Dias, 2010).

A partir de la década de 1970, el término competencia se associa con la calificación profesional. Más precisamente, en 1973 David McClelland, con la publicación de su estudio Testing for competence rather than intelligence, definió el concepto de competencias como rasgos de personalidad o características individuales, esenciales para el desempeño superior de los empleados en la realización de tareas (Fleury \& Fleury, 2001; Skorková, 2016).

De esta manera, la noción de competencia se ha utilizado comúnmente para referirse a la capacidad necesaria para realizar una determinada actividad, o sea, al saber cómo hacer, para poner en práctica los conocimientos adquiridos (Setlhako, 2014; Tenório et al., 2016; Skorková, 2016). Para Fleury y Fleury (2001, p. 188), competencia es "saber cómo actuar de manera responsable y reconocida, lo que implica movilizar, integrar, transferir conocimientos, recursos y habilidades, que agregan valor económico a la organización y valor social al individuo", denotando, de esta manera, su contribución personal y para la efectividad de las acciones en el centro de las actividades profesionales.

Considerando las definiciones, existe un consenso entre los autores de que la competencia consiste básicamente en tres elementos, a saber: conocimiento, habilidades y actitudes. El conocimiento consiste en un conjunto de información útil y necesaria para el desempeño de tareas y la solución de problemas inherentes a la función. Las habilidades se relacionan con la aplicación de prácticas para hacer el trabajo correctamente, incluyendo, por ejemplo, pensamiento lógico y buena comunicación. A su vez, las actitudes se refieren a las maneras en que el profesional aplica sus valores, principios y creencias en forma de conducta en el entorno laboral (Sousa \& Barbosa, 2018).

Para Marinho-Araújo y Almeida (2016), una competencia se construye en la vida cotidiana sociocultural y en situaciones relacionales entre individuos que requieren una postura activa frente a desafíos y problemas. Ser competente, por lo tanto, implica la movilización de los recursos disponibles (conocimiento, habilidades, creencias, actitudes, principios, etc.), lo que permite tomar decisiones y referencias apropiadas para enfrentar la situación problemática que se presenta. Por lo tanto, desde el punto de vista de los autores, el desarrollo de competencias es un proceso largo, ya que implica la construcción de una historia profesional que asocie características personales y la función desempeñada, lo que requiere el desarrollo de competências que articulen teorías, métodos y experiencias para identificar y resolver problemas en el contexto social y profesional.

Se pueden desarrollar varias competencias para apoyar el desempeño de actividades/funciones. En este sentido, Grzybowka y Lupicka (2017) presentan tres categorías principales que serán utilizadas para la clasificación de competencias en este estudio. El primero consiste en competencias técnicas que incluyen conocimientos y

El papel del tutor en la enseñanza de la educación a distancia: una revisión sistemática sobre el enfoque de habilidades. Massuga, Soares y Doliveira.

Página 6 de 26 
habilidades relacionadas con el trabajo, adquiridas a través de la práctica y el aprendizaje. El segundo grupo está formado por competencias gerenciales, que abordan las habilidades para la resolución de problemas y la toma de decisiones. Finalmente, destacan las competencias sociales que incluyen los valores y motivaciones sociales de un individuo, siendo la base para la interacción con los demás.

Sobre el tema, Borges et al. (2014), entienden la necesidad de diferenciar las competencias técnicas y de comportamiento. En opinión de los autores, las competencias técnicas corresponden a conocimientos específicos y habilidades de una función, o sea, están cerca del puesto del empleado. Las competencias conductuales se basan en actitudes y se consideran cercanas a las personas, expresando sus características individuales con mayor fuerza.

A su vez, de forma más completa, Resende (2000) clasificó las competencias en nueve categorías, siendo: 1. Competencias técnicas: relacionadas con el conocimiento especializado dirigido a un determinado tema o función; 2. Competencias intelectuales referidas a las aptitudes mentales; 3 . Competencias cognitivas: combinación de capacidad intelectual y dominio del conocimiento; 4. Competencias relacionales: capacidad de interactuar con otros; 5. Competencias sociales y políticas: capacidad de actuar y participar en eventos sociales y políticos; 6. Competencias didáctico-pedagógicas: dirigidas a la educación y la enseñanza; 7. Competencias metodológicas: capacidad para aplicar técnicas de organización del trabajo; 8. Competencias de liderazgo: capacidad de influir y dirigir a las personas; y 9. Competencias comerciales: relacionadas con el establecimiento y la búsqueda de objetivos en la gestión organizacional.

En resumen, el enfoque de competencia se ha utilizado y discutido en el centro de la calificación profesional, siendo una construcción formada por conocimientos, habilidades y actitudes esenciales para el desempeño de una tarea o actividad. Existen varios tipos y clasificaciones de competencias que son específicas de cada área de especialización, incluidas las funciones de tutoría en educación a distancia. En este contexto específico, se pretende, por tanto, comprender cuáles son las competencias técnicas, gerenciales y sociales intrínsecas el papel de tutor, así como, los aspectos más destacados y dificultades aún evidentes en el ejercicio de la función.

\section{Método}

Con el fin de identificar las competencias relacionadas con el tutor de educación a distancia y lograr los objetivos subyacentes, se realizó una investigación con enfoque cualitativo/descriptivo. Según Richardson (2010), la investigación cualitativa apunta a situaciones complejas y particulares, posibilitando el análisis del problema, sus interacciones y procesos y la comprensión en mayor profundidad del objeto sin el uso de herramientas estadísticas. A su vez, la investigación descriptiva tiende a examinar un fenómeno describiendo sus características, naturaleza, causa, frecuencia y conexiones (Barros \& Lehfeld, 2007).

En cuanto a los procedimientos técnicos, se decidió realizar una revisión sistemática de la literatura en bases de datos con contenido de gran alcance. De acuerdo con Denyer y Tranfield (2009), la revisión sistemática consiste en una metodología que localiza los estudios existentes, selecciona y evalúa sus contribuciones, sintetizando datos con el fin de aportar conclusiones sobre un tema específico. Se diferencia de una revisión

El papel del tutor en la enseñanza de la educación a distancia: una revisión sistemática sobre el enfoque de habilidades. Massuga, Soares y Doliveira.

Página 7 de 26 
de la literatura tradicional porque consiste en un proyecto de investigación independiente que explora un problema que se evalúa utilizando criterios exigentes y claramente definidos.

Para este estudio en particular, se adoptó la metodología Methodi Ordinatio para la selección y evaluación de la calidad de la literatura cubierta. El método utiliza la ecuación InOrdinatio para clasificar los estudios considerando su relevancia en función del factor de impacto de la revista, el número de citas y el año de publicación del artículo. Además, se divide en 9 etapas, siendo: I - Establecimiento del objetivo de investigación; II - Investigación preliminar con las palabras clave en las bases de datos; III - Elección definitiva de la combinación de palabras clave, bases de datos y delimitación de tiempo; IV - Búsqueda efectiva en las bases de datos; V - Aplicación de procedimientos de filtrado; VI - Identificación del factor de impacto, año de publicación y número de citas; VII - Clasificación de artículos usando InOrdinatio; VIII - localización de estudios en formato completo; y IX - Lectura y análisis sistemática de las obras (Pagani, Kovaleski $\&$ Resende, 2015). Estos pasos pueden ser mejor vistos y comprendidos en la Figura siguiente:

El papel del tutor en la enseñanza de la educación a distancia: una revisión sistemática sobre el enfoque de habilidades. Massuga, Soares y Doliveira. 
Figura 1 - Representación gráfica de la metodologia Methodi Ordinatio

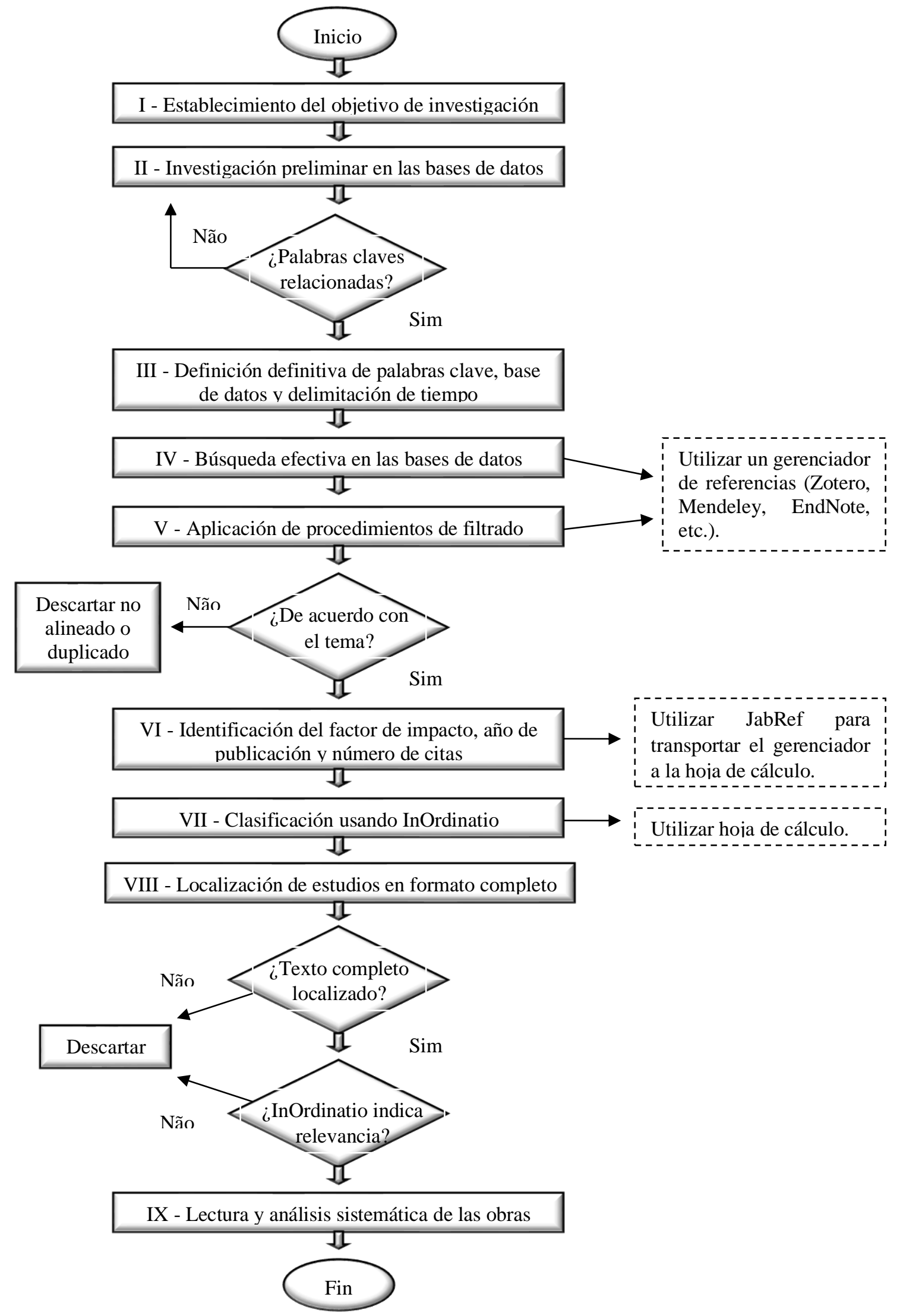

Fonte: Adaptado de Pagani et al. (2015) y Pagani, Kovaleski \& Resende (2017).

El papel del tutor en la enseñanza de la educación a distancia: una revisión sistemática sobre el enfoque de habilidades. Massuga, Soares y Doliveira. 
Después de definir la intención del estudio, se realizó una investigación exploratoria en las bases de datos del Portal de Revistas Capes; Biblioteca electrónica científica en línea (SCIELO); ScienceDirect y Scopus. La elección de varias fuentes de búsqueda se justifica según la perspectiva de Donato y Donato (2019), quienes mencionan la necesidad de utilizar en una revisión sistemática al menos tres bases de datos con el fin de incluir todo el contenido correspondiente a la temática definida.

Para realizar la investigación se utilizaron las siguientes palabras clave directamente relacionadas con el tema tratado: tutoria; tutor; ensino a distância, EAD, competências, mentoring, tutor, tutoring, distance learning, E-learning, skills y competencies. La descripción booleana se utilizó para combinar los términos seleccionados. Con el fin de aumentar la sensibilidad de la investigación, solo se cubrieron artículos de investigación y revisión de los últimos 10 años (2010-2020) y se aplicaron los filtros por título, tema, resumen y palabras clave.

La búsqueda preliminar realizada el 5 de junio de 2020 resultó en un total de 166 estudios, de acuerdo con el procedimiento y la distribución que se muestran en lo Cuadro a continuación:

Cuadro 1 - Selección preliminar de estudios según procedimientos de búsqueda

\begin{tabular}{|c|c|c|c|c|}
\hline $\begin{array}{l}\text { BASE DE } \\
\text { DATOS }\end{array}$ & $\begin{array}{c}\text { DESCRIPTORES Y } \\
\text { OPERADORES } \\
\text { BOOLEANOS } \\
\end{array}$ & $\begin{array}{l}\text { RESTRICCIONES DE } \\
\text { INVESTIGACIÓN }\end{array}$ & $\begin{array}{c}\text { FECHA } \\
\text { ACCESO }\end{array}$ & $\begin{array}{c}\text { RESULTADOS } \\
\text { DE LA } \\
\text { BÚSQUEDA } \\
\end{array}$ \\
\hline $\begin{array}{c}\text { Portal de } \\
\text { Revistas Capes }\end{array}$ & \multirow{4}{*}{$\begin{array}{c}\text { 1- (Tutoria OR tutor) } \\
\text { AND (ensino a } \\
\text { distância OR EAD) } \\
\text { AND competências } \\
2 \text { - (Mentoring OR } \\
\text { tutor OR tutoring) } \\
\text { AND (distance learning } \\
\text { OR E-learning) AND } \\
\text { (skills OR } \\
\text { competencies) } \\
\end{array}$} & \multirow{4}{*}{$\begin{array}{c}\text { - Límite de tiempo: } \\
2010 \text {-2020 } \\
\text { - Título, tema, resumen } \\
\text { y palabras clave } \\
\text { - Artículos de } \\
\text { investigación o revisión }\end{array}$} & \multirow{4}{*}{$15 / 06 / 2020$} & 17 \\
\hline SCIELO & & & & 8 \\
\hline ScienceDirect & & & & 44 \\
\hline Scopus & & & & 97 \\
\hline \multicolumn{4}{|c|}{ TOTAL } & 166 \\
\hline
\end{tabular}

Fuente: Datos de investigación (2020).

Debido a la posibilidad de incluir en el portafolio trabajos de áreas no relacionadas, se adoptaron procedimientos de filtrado que consisten en eliminar artículos duplicados y analizar los títulos, resúmenes y palabras clave Con la ayuda del gerenciador de referencia Zotero, se eliminaron 11 artículos por duplicado, quedando 155 para su posterior análisis, considerando los siguientes criterios:

- Criterios electivos: a) trabajos directamente relacionados con la descripción de las competencias de los tutores en educación a distancia; b) estudios completos publicados en revistas.

- Criterios de exclusión: a) artículos publicados en eventos científicos; b) trabajos no relacionados con el tema; c) estudios que aborden las competencias desde la perspectiva del estudiante; d) artículos que se centran en el análisis de herramientas para la evaluación de las competencias docentes o estudiantiles; e) trabajos que abordan la tutoría en cursos presenciales, fundamentalmente aquellos enfocados al área de la salud.

El papel del tutor en la enseñanza de la educación a distancia: una revisión sistemática sobre el enfoque de habilidades. Massuga, Soares y Doliveira. 
Después del filtrado, 12 artículos cumplieron los criterios definidos y se sometieron a un análisis de la calidad de la literatura seleccionada. En este paso, se utilizó el JabRef para enviar los datos del gerenciador de referencias a una hoja de cálculo. En primer lugar, en una hoja de cálculo de Excel, se insertaron los datos del factor de impacto de las revistas a las que son sometidos los artículos utilizando la última clasificación de lo Journal Citation Reports (JCR 2020, año base 2019), el año de publicación y el número de citas que se detallan en la plataforma de Google Académico. Luego, estos datos se sometieron a la siguiente ecuación:

$$
\text { InOrdinatio }=(\mathrm{Fi} / 1000)+\alpha^{*}[10-(\text { AnoPesq }- \text { AnoPub })]+\left(\sum \mathrm{Ci}\right)
$$

Dónde:

$F i$ : factor de impacto;

$\alpha$ : coeficiente atribuido por el investigador a la relevancia del año de publicación, que puede variar de 1 a 10;

AnoPesq: año de investigación;

AnoPub: año de publicación;

$C i$ : Número de citas.

Una vez procesados los datos, se obtiene el InOrdinatio de cada artículo, y cuanto mayor es el índice, más relevantes es el artículo evaluado (Pagani et al., 2015). Para este estudio en particular, se asignó un valor de 5 para el coeficiente $\alpha$, teniendo en cuenta que la relevancia del período de publicación ya se ha considerado parcialmente al delimitar temporalmente la investigación (últimos 10 años). En función de los resultados obtenidos, se incluyeron los artículos con puntuaciones positivas superiores a veinte (>20). En este caso, todos los estudios cumplieron con los criterios y fueron ubicados y considerados para lectura final y análisis sistemática. La siguiente Figura muestra el proceso metodológico llevado a cabo para obtener el portafolio bibliográfico final:

El papel del tutor en la enseñanza de la educación a distancia: una revisión sistemática sobre el enfoque de habilidades. Massuga, Soares y Doliveira. 
Figura 2 - Proceso metodológico de construcción del portafolio bibliográfico.

\section{Estudios identificados después de} buscar en las bases de datos

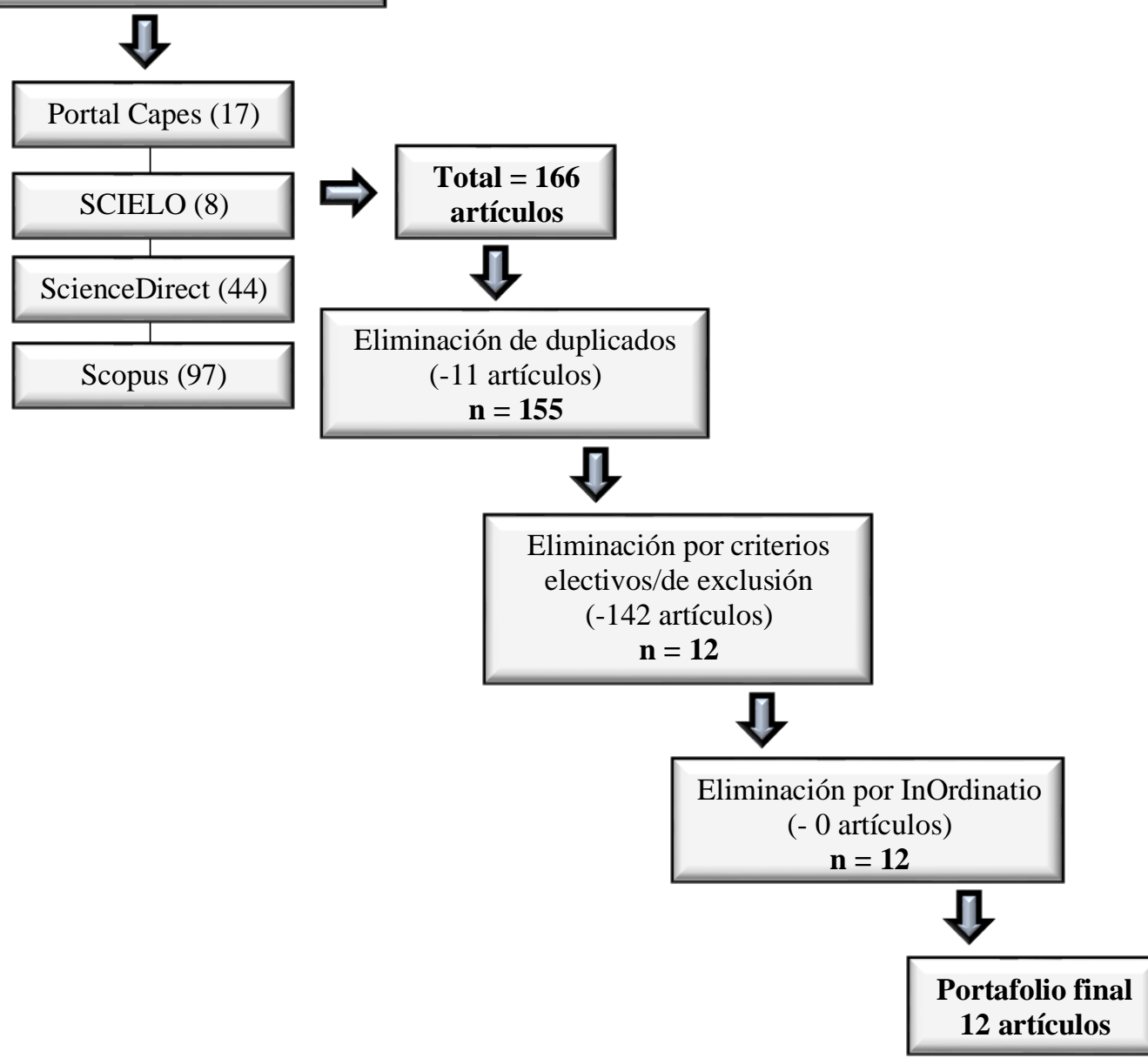

Fuente: Datos de investigación (2020).

En los resultados y discusiones se detallan las principales evidencias presentadas en los estudios que componen el portafolio final, orientando la comprensión relacionada con las competencias de tutoría en educación a distancia, de acuerdo con los objetivos establecidos.

\section{Resultados y discusiones}

Considerando los criterios establecidos, se cubrieron 12 estudios para el análisis final. En la siguiente tabla se encuentran las especificaciones de los artículos considerando la clasificación obtenida por el método InOrdinatio. 
Tabla 1 - Artículos seleccionados y ordenados según InOrdinatio

\begin{tabular}{|c|c|c|c|}
\hline $\mathrm{n}^{\mathbf{0}}$ & Autor(es) & Título & InOrdinatio \\
\hline 1 & $\begin{array}{l}\text { Hrastinski et al. } \\
(2018)\end{array}$ & $\begin{array}{l}\text { Tutoring online tutors: using digital badges to encourage } \\
\text { the development of online tutoring skills }\end{array}$ & 52,951 \\
\hline 2 & Adnan (2018) & $\begin{array}{l}\text { Professional development in the transition to online } \\
\text { teaching: the voice of entrant online instructors }\end{array}$ & 50,842 \\
\hline 3 & $\begin{array}{l}\text { Metz } \quad y \\
\text { Bezuidenhout } \\
(2018)\end{array}$ & $\begin{array}{l}\text { An importance-competence analysis of the roles and } \\
\text { competencies of e-tutors at an open distance learning } \\
\text { institution }\end{array}$ & 49,956 \\
\hline 4 & Kara y Can (2019) & $\begin{array}{l}\text { Master's students' perceptions and expectations of good } \\
\text { tutors and advisors in distance education }\end{array}$ & 48,297 \\
\hline 5 & $\begin{array}{l}\text { Campbell et al., } \\
\text { (2019) }\end{array}$ & $\begin{array}{l}\text { The perceptions of STEM tutors on the role of tutorials in } \\
\text { distance learning }\end{array}$ & 46 \\
\hline 6 & $\begin{array}{l}\text { Li, Zhang, Yu y } \\
\text { Chen (2017) }\end{array}$ & $\begin{array}{l}\text { Rethinking distance tutoring in e-learning environments: } \\
\text { A study of the priority of roles and competencies of open } \\
\text { university tutors in China }\end{array}$ & 45,297 \\
\hline 7 & 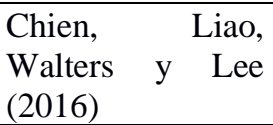 & $\begin{array}{l}\text { Measuring the Moral Reasoning Competencies of } \\
\text { Service-Learning e-Tutors }\end{array}$ & 42 \\
\hline 8 & $\begin{array}{l}\text { Murphy, Shelley, } \\
\text { White y Baumann } \\
\text { (2011) }\end{array}$ & $\begin{array}{l}\text { Tutor and student perceptions of what makes an effective } \\
\text { distance language teacher }\end{array}$ & 39,702 \\
\hline 9 & $\begin{array}{l}\text { Tenório et al. } \\
\text { (2016) }\end{array}$ & $\begin{array}{l}\text { Levantamento de competências pedagógicas necessárias a } \\
\text { tutores da educação a distância }\end{array}$ & 35 \\
\hline 10 & $\begin{array}{l}\text { Borges et al., } \\
(2014)\end{array}$ & $\begin{array}{l}\text { Diagnóstico de competências individuais de tutores que } \\
\text { atuam na modalidade a distância }\end{array}$ & 30 \\
\hline 11 & $\begin{array}{l}\text { Murphy, Shelley y } \\
\text { Baumann (2010) }\end{array}$ & $\begin{array}{l}\text { Qualities of effective tutors in distance language teaching: } \\
\text { Student perceptions }\end{array}$ & 23 \\
\hline 12 & Setlhako (2014) & $\begin{array}{l}\text { Competencies required for the facilitation of online } \\
\text { learning courses: An online teaching assistants } \\
\text { competency based approach }\end{array}$ & 21 \\
\hline
\end{tabular}

Fuente: Datos de investigación (2020).

El concepto de competencias es visto como una alternativa a la capacidad, habilidad, aptitud, potencial y conocimiento. Son las competencias las que hacen que el sujeto afronte y regule adecuadamente un conjunto de tareas y funciones predeterminadas (Dias, 2010). El tutor en educación a distancia, tiene una diversidad de roles que se distinguen de los que se aplican en el aula en la modalidad presencial, por lo tanto, se necesitan diferentes competencias para realizar su trabajo de manera satisfactoria (Kopp et al., 2012; Setlhako, 2014).

Dados los trabajos seleccionados, se discuten varias competencias considerando el papel del tutor en la educación a distancia. Hrastinski et al. (2018), para comprender cómo las insignias digitales podrían fomentar el desarrollo de competencias de tutoría en línea, consideraron tres competencias principales: alentar la discusión para facilitar el aprendizaje, alentar la reflexión de lo aprendido y brindar apoyo emocional. 13 tutores en matemáticas de primaria y secundaria debería enviar un fragmento de una conversación para ilustrar cada competencia. Los resultados muestran la importancia de reflexionar sobre las prácticas y habilidades de tutoría a través de insignias digitales. Con respecto a las competencias, fue posible observar que todos los tutores practicaron con frecuencia la competencia para incentivar la discusión, seguida de la competencia para fomentar la reflexión, informada por 9 de los tutores. Finalmente, brindar apoyo emocional fue presentado por 8 tutores, siendo una competencia considerada desafiante.

El papel del tutor en la enseñanza de la educación a distancia: una revisión sistemática sobre el enfoque de habilidades. Massuga, Soares y Doliveira. 
Adnan (2018) buscó, a través de su estudio, examinar un programa en línea de desarrollo de profesores de idiomas en Turquía. Las reflexiones de los participantes muestran que se requieren competencias para los entornos de aprendizaje en línea con el fin de mejorar los roles tradicionales de la educación a distancia. Entre los objetivos, el autor buscó evaluar las habilidades percibidas por los profesores de idiomas en la enseñanza en línea. 37 participantes respondieron a la investigacíon. Los resultados muestran que los participantes no están seguros de su nivel de competencia para la enseñanza en línea. Las competencias de mayor puntaje consisten en: comunicarse de manera efectiva con los estudiantes y proporcionar retroalimentación eficiente a los estudiantes. Las habilidades intermedias incluyen: usar herramientas de comunicación, diseñar actividades de aprendizaje, actividades de evaluación y consideraciones éticas. Las competencias con la puntuación más baja son: desarrollo de contenido compacto del curso electrónico en diferentes formatos, creación de contenido en un formato diferente del curso electrónico (texto, audio, video) y uso efectivo del sistema y gestión del aula virtual, es decir, hay una mayor dificultad cuando la tecnología se incluye en las tareas.

El estudio de Metz y Bezuidenhout (2018) buscó investigar el papel del tutor electrónico del curso de Ciencias Económicas y Administrativas en una Universidad de Sudáfrica, en términos de percepciones de sus roles y la evaluación de sus competencias. En total, se investigaron 164 e-tutores. Las competencias cubiertas se dividen en tres sectores, a saber:

- Compromiso social: facilitar la comprensión del contenido por parte de los estudiantes, apoyar la reflexión de las actividades y resultados de aprendizaje, proporcionar un entorno en línea acogedor, gestionar la comunicación y las discusiones en línea, proporcionar realimentación oportuna, acompañar al alumno durante todo el proceso.

- Administración del entorno en línea: ayudar a los estudiantes a desarrollar habilidades, tomar decisiones tecnológicas para el entorno en línea, repasar tareas, administrar y mantener registros, establecer reglas básicas.

- Desarrollo y creación de material de apoyo: ser un ejemplo de comportamiento social en línea, establecer su propia identidad, desarrollar recursos para apoyar el aprendizaje, crear nuevos conocimientos relevantes para el contenido.

Se observó que existen varias brechas entre las competencias y los roles que esperan cumplir, con diferencias más notables en el factor de compromiso social. Simplemente no hay brechas de competencia en términos de gestión, administración y mantenimiento de registros, establecer reglas básicas, ser un ejemplo de comportamiento social en línea, establecer su propia identidad, acompañar al estudiante durante todo el proceso y proporcionar realimentación oportuna.

De manera diferente, el estudio de Kara y Can (2019) tuvo como objetivo explorar las percepciones y expectativas de los estudiantes de maestría sobre el perfil de buenos tutores en los programas de educación a distancia. Se evaluaron 143 estudiantes de 4 programas en dos universidades de Turquía. Los resultados revelan que los buenos tutores proporcionan un ambiente de aprendizaje estimulante centrado en el estudiante, tienen una interacción y comunicación individualizada y atenta, tienen experiencia en el tema y habilidades básicas en tecnología.

El papel del tutor en la enseñanza de la educación a distancia: una revisión sistemática sobre el enfoque de habilidades. Massuga, Soares y Doliveira. 
Campbell et al. (2019), a su vez, buscó investigar el modelo de aprendizaje a distancia de la Universidad Abierta del Reino Unido a través de entrevistas con 19 tutores para comprender su percepción sobre el papel y los objetivos de los tutoriales y sobre las expectativas de los estudiantes en relación con las clases grupales. En cuanto al papel del tutor en las clases grupales, se destacan las competencias de facilitar el aprendizaje y las habilidades académicas, apoyar la construcción de confianza, motivación, interacción social y habilidades para trabajar en un grupo colaborativo. Entre las dificultades reportadas, se destaca el cambio a la enseñanza en línea utilizando sistemas sincrónicos que presentan un desafío en la creación de interacción entre los estudiantes.

El estudio de Li et al. (2017), buscó identificar la prioridad de los roles y competencias de los tutores para el cambio a la educación a distancia en línea en China. En el estudio, se identificaron siete roles (instructor, diseñador instruccional, facilitador/consultor de aprendizaje, social, evaluador, tecnólogo y gerente/administrador). Según los roles, se reconocieron 98 tareas críticas. Las competencias requeridas para realizar las 98 tareas se agruparon en 20 categorías, que están asociadas con los 7 roles, como se muestra en lo Cuadro 2 a continuación:

Cuadro 2 - Roles y habilidades del tutor según un estudio de Li et al. (2017)

\begin{tabular}{|c|l|}
\hline ROLES & \multicolumn{1}{|c|}{ HABILIDAD } \\
\hline Instructor & $\begin{array}{l}\text { Facilitar el proceso cognitivo del contenido del curso; organizar y guiar el } \\
\text { experimento o practicar actividades en el curso; desarrollar recursos de } \\
\text { aprendizaje del curso; facilitar la interacción productiva interpersonal en el } \\
\text { curso. }\end{array}$ \\
\hline Diseñador instruccional & $\begin{array}{l}\text { Desarrollar el plan de tutoría para el curso para estudiantes locales; crear } \\
\text { actividades de interacción para un aprendizaje eficaz. }\end{array}$ \\
\hline $\begin{array}{c}\text { Facilitador de } \\
\text { aprendizaje/consultor }\end{array}$ & $\begin{array}{l}\text { Motivar a los estudiantes; facilitar el aprendizaje grupal; desarrollar la } \\
\text { capacidad de aprendizaje autorregulada de los estudiantes; ofrecer consejos } \\
\text { y sugerencias basados en las necesidades de los estudiantes. }\end{array}$ \\
\hline Tecnólogo & $\begin{array}{l}\text { Usar tecnologías para facilitar y mejorar la enseñanza y el aprendizaje; } \\
\text { ayudar a los estudiantes a resolver problemas tecnológicos básicos } \\
\text { encontrados en el proceso de aprendizaje. }\end{array}$ \\
\hline Social & $\begin{array}{l}\text { Construir y mantener un ambiente de aprendizaje cordial; resolver conflictos } \\
\text { estudiantiles amigablemente; promover la construcción y el desarrollo de } \\
\text { una comunidad de aprendizaje. }\end{array}$ \\
\hline Evaluador & $\begin{array}{l}\text { Evaluar el rendimiento de aprendizaje de los estudiantes en el curso; evaluar } \\
\text { el curso. }\end{array}$ \\
\hline Gerente/Administrador & $\begin{array}{l}\text { Monitorear el progreso individual y grupal; gestionar el progreso de las } \\
\text { actividades de interacción educativa; regular el comportamiento del alumno. }\end{array}$ \\
\hline
\end{tabular}

Fuente: Adaptado de Li et al. (2017)

La prioridad de roles y competencias se evaluó de acuerdo con un cuestionario aplicado a tutores, estudiantes y administradores. La mayoría de las competencias con los puntajes de mayor importancia se asociaron con los roles de instructor, evaluador y diseñador instruccional. La competencia "facilitar el proceso cognitivo del contenido del curso", obtuvo la máxima puntuación. Competencias: motivar a los estudiantes, facilitar la interacción interpersonal y construir y mantener un ambiente de aprendizaje cordial se encuentran entre las 10 competencias principales, lo que lleva a la necesidad de una mayor atención a las competencias que facilitan la participación de los estudiantes en el aprendizaje. Además, se enfatizaron las habilidades de los tutores para mejorar la interacción personal. En general, los autores entienden que la prioridad de los roles y las

El papel del tutor en la enseñanza de la educación a distancia: una revisión sistemática sobre el enfoque de habilidades. Massuga, Soares y Doliveira. 
competencias cambia cuando se trata de la educación a distancia, pasando de una perspectiva de la pedagogía cognitivo-conductual al constructivista social y conectivista.

Considerando el desarrollo moral de los tutores, Chien et al., (2016), exploran, en su estudio, el desarrollo de competencias de razonamiento moral por parte de 15 estudiantes universitarios que desempeñaban el papel de tutores en línea para estudiantes de primaria de áreas remotas. Se reconocen cinco competencias, a saber: carácter moral, resolución de problemas, afecto, empatía e interacción social. La investigacíon también señaló que la interacción social durante las clases beneficiaba las habilidades de comunicación de los tutores, reduciendo el contenido y la ansiedad. La empatía estuvo presente en la tutoría electrónica, y los tutores también demostraron consideraciones en relación con la vida diaria.

Sobre el tema, Murphy et al., (2011), buscaron determinar las actitudes, habilidades y conocimientos que necesitan los tutores de lenguaje a distancia, en base a la percepción de tutores y estudiantes. El estudio se realizó con tutores y estudiantes de cursos de francés, alemán y español en The Open University en el Reino Unido, a través de cuestionarios y, posteriormente, entrevistas en profundidad. El estudio revela algunas competencias para el desarrollo de tutores del lenguaje en la educación a distancia: la articulación de la comunicación no verbal; empatía con la experiencia del alumno incluidos factores de motivación, diferenciación de la enseñanza para satisfacer las necesidades individuales, atención a problemas efectivos y cualidades personales necesarias.

Tenório et al. (2016) tuvieron como objetivo presentar la percepción de 10 tutores de cursos de educación a distancia en Rio de Janeiro sobre las habilidades pedagógicas esenciales, la importancia que se les atribuye y las dificultades para aplicarlas. Entre las habilidades consideradas esenciales por los autores estaban: motivar el aprendizaje, usar un lenguaje claro y amigable, aceptar la heterogeneidad del conocimiento de los estudiantes, estimular la capacidad crítica, evaluar el desarrollo y proporcionar retroalimentación de las tareas. Los resultados demuestran que los tutores tenían las habilidades pedagógicas vitales y las mejoraron, a través de la actualización profesional, pero no todos mantuvieron una capacitación continua. Para los entrevistados, la competencia que se considera más importante sería la capacidad de desarrollar la capacidad crítica de los estudiantes, pero creían que eran mejores para motivar el aprendizaje, ganar empatía, usar un lenguaje clara y amigable y proporcionar retroalimentación. Las competencias consideradas como las más importantes se señalaron como aquellas sobre las que los tutores tenían menos control. Como la competencia más difícil de aplicar en la opinión de los encuestados es evaluar el aprendizaje, debido a la necesidad de criterios objetivos y al mismo tiempo subjetivos al considerar las particularidades de cada alumno.

El estudio de Borges et al. (2014), buscó realizar un diagnóstico de competencias individuales, técnicas y de comportamiento, necesarias como tutor en la educación a distancia dentro del alcance de la Universidad Abierta de Brasil (UAB), así como identificar los grados de dominio e importancia y la brecha entre estos factores. El estudio consta de dos fases. La fase 1 es cualitativa y consistió en un análisis documental, 17 entrevistas individuales y 2 grupos focales. En esta etapa, se plantearon las habilidades que formaban parte del cuestionario. Entre ellos, se destacan el dominio del contenido, aspectos relacionados con el funcionamiento del entorno virtual y otras herramientas que optimizan e interactúan con los alumnos, en el caso de las habilidades técnicas. En

El papel del tutor en la enseñanza de la educación a distancia: una revisión sistemática sobre el enfoque de habilidades. Massuga, Soares y Doliveira.

Página 16 de 26 
relación con las habilidades de comportamiento, se evidencia la motivación del estudiante, la individualización del servicio, la cordialidad, la empatía, la persuasión, la capacidad de trabajar en equipo, el compromiso y la capacidad de planificar y organizar. El segundo constituyó la fase cuantitativa con la aplicación de 200 cuestionarios, en los cuales se encontró que las brechas eran bajas, lo que indica que los tutores tienen experiencia en su actuación. Entre las competencias con la mayor brecha, está la capacidad del tutor para intervenir en el ambiente de aprendizaje virtual y otras 8 de naturaleza técnica. Las competencias conductuales, a su vez, se desarrollan parcial o totalmente entre los tutores investigados.

Similar al estudio de Murphy et al. (2011), la investigación realizada por Murphy et al. (2010), buscó dirigir la atención a las competencias necesarias para la enseñanza efectiva de idiomas a distancia centrada en la percepción del alumno. Se realizó un estudio con 144 estudiantes de cursos de francés, alemán y español en The Open Universtity en el Reino Unido. También se realizaron entrevistas con 12 estudiantes para reforzar la información. En opinión de los estudiantes, la tutoría efectiva se relaciona con tres meta temas, a saber: dominios de la experiencia del tutor; dimensiones afectivas de la participación del tutor y las dimensiones organizativas de la práctica del tutor. Con respecto a la experiencia del tutor, los estudiantes valoran a los tutores capaces de proporcionar ejemplos significativos, accesibles y útiles y con conocimiento sobre el tema, además, valoran la competencia de los hablantes nativos, la experiencia docente y un buen conocimiento del curso. Con respecto a las dimensiones afectivas de la participación, los estudiantes esperan tutores que sean sensibles a sus necesidades y aliento, al mismo tiempo que valoran a tutores que establecen una atmósfera amigable, que son entusiastas, accesibles, solidarios y comprometidos y que pueden responder a las necesidades individuales. En cuanto a las dimensiones organizativas, se destacan las competencias organizativas y de enfoque para que los tutores hagan un mejor uso del tiempo de contacto y las competencias de TI y los sistemas organizativos de apoyo al estudiante.

Finalmente, Setlhako (2014) buscó, a través de un estudio cualitativo, comprender los roles y competencias de 11 tutores a distancia de la Universidad de Sudáfrica, desarrollados antes y después de su desempeño como tales, también considerando aquellos que necesitan ser adquiridos. Teniendo en cuenta las habilidades obtenidas antes de trabajar en la tutoría en línea, se destacan los conocimientos informáticos y otras habilidades técnicas para operar una computadora. Solo un tutor ya tenía conocimiento sobre el ambiente de aprendizaje virtual. Después de actuar como tutores, los participantes ganaron confianza en las habilidades técnicas y pedagógicas, como usar el software de atribución (jRouter), mantener un contacto constante con estudiantes y profesores, respetar el aprendizaje diverso, la capacidad de gestionar la interacción de los estudiantes y liderar y controle de las discusiones en línea. Los encuestados también informaron la necesidad de desarrollar competencias para proporcionar retroalimentación a todos los estudiantes individualmente y para llegar a los estudiantes que no interactúan.

Teniendo en cuenta los resultados presentados, lo siguiente Cuadro identifica las principales competencias necesarias para que el tutor actúe en la educación a distancia, adoptando la clasificación de Grzybowka y Lupicka (2017). Además, la Figura 03 de la secuencia ilustra las mismas competencias considerando la representación según el número de citas.

El papel del tutor en la enseñanza de la educación a distancia: una revisión sistemática sobre el enfoque de habilidades. Massuga, Soares y Doliveira.

Página 17 de 26 
Cuadro 3 - Competencias asociadas al desempeño del tutor en educación a distancia, según estudios evaluados.

\begin{tabular}{|c|c|c|}
\hline $\begin{array}{c}\text { GRUPO DE } \\
\text { COMPETENCIAS }\end{array}$ & AUTOR(ES) & COMPETENCIAS CUBIERTAS \\
\hline \multirow{14}{*}{$\begin{array}{l}\text { COMPETENCIAS } \\
\text { TÉCNICAS }\end{array}$} & $\begin{array}{l}\text { Setlhako (2014); Tenório et al. } \\
\text { (2016); Adnan (2018); Metz y } \\
\text { Bezuidenhout (2018) }\end{array}$ & Proporcionar realimentación \\
\hline & Adnan (2018) & Usar herramientas de comunicación \\
\hline & $\begin{array}{l}\text { Li et al. (2017); Adnan (2018); } \\
\text { Metz y Bezuidenhout (2018) }\end{array}$ & $\begin{array}{l}\text { Desarrollar contenidos y actividades de } \\
\text { aprendizaje }\end{array}$ \\
\hline & $\begin{array}{l}\text { Tenório et al. (2016); Li et al., } \\
\text { (2017); Adnan (2018) }\end{array}$ & Habilidades de evaluación del alumno \\
\hline & Li et al., (2017) & Evaluación del curso \\
\hline & $\begin{array}{l}\text { Murphy et al. (2010); Borges et al. } \\
\text { (2014); Setlhako (2014); Li et al. } \\
\text { (2017); Adnan (2018); Kara y Can } \\
\text { (2019) }\end{array}$ & $\begin{array}{l}\text { Uso del sistema y conocimiento en } \\
\text { Tecnología de la Información (TI) }\end{array}$ \\
\hline & Metz y Bezuidenhout (2018) & Revisar tareas/contenido/actividades \\
\hline & Metz y Bezuidenhout (2018) & Mediar comunicación y debates en línea \\
\hline & $\begin{array}{l}\text { Li et al. (2017); Metz } \quad \text { y } \\
\text { Bezuidenhout (2018); Campbell } \\
\text { al. (2019) }\end{array}$ & $\begin{array}{l}\text { Facilitar el aprendizaje, el desarrollo de } \\
\text { habilidades y la comprensión del } \\
\text { contenido por parte de los alumnos }\end{array}$ \\
\hline & $\begin{array}{l}\text { Murphy et al. (2010); Borges et al. } \\
\text { (2014); Kara y Can (2019) }\end{array}$ & $\begin{array}{l}\text { Experiencia en el tema y dominio de } \\
\text { contenido }\end{array}$ \\
\hline & Li et al. (2017) & Guiar las actividades del curso \\
\hline & Li et al. (2017) & Facilitar el aprendizaje grupal \\
\hline & Li et al. (2017) & $\begin{array}{l}\text { Ayudar a los estudiantes a resolver } \\
\text { problemas tecnológicos }\end{array}$ \\
\hline & Tenório et al. (2016) & Estimular la capacidad crítica del alumno \\
\hline \multirow{9}{*}{$\begin{array}{l}\text { COMPETENCIAS DE } \\
\text { GESTIÓN }\end{array}$} & Adnan (2018) & Gestión del aula virtual \\
\hline & $\begin{array}{l}\text { Li et al. (2017); Setlhako (2014); } \\
\text { Metz y Bezuidenhout (2018) }\end{array}$ & $\begin{array}{l}\text { Acompañar al alumno durante todo el } \\
\text { proceso }\end{array}$ \\
\hline & Metz y Bezuidenhout (2018) & $\begin{array}{l}\text { Tomar decisiones tecnológicas para el } \\
\text { entorno en línea }\end{array}$ \\
\hline & Metz y Bezuidenhout (2018) & Administrar y mantener registros \\
\hline & Metz y Bezuidenhout (2018) & Establecer reglas básicas \\
\hline & Li et al. (2017) & Desarrollar un plan de curso tutorial. \\
\hline & Chien et al. (2016) & Solución de problemas \\
\hline & $\begin{array}{l}\text { Murphy et al. (2010); Borges et al. } \\
\text { (2014) }\end{array}$ & $\begin{array}{llll}\begin{array}{l}\text { Habilidades } \\
\text { organización }\end{array} & \text { de } & \text { planificación } & y \\
\end{array}$ \\
\hline & Setlhako (2014) & Dirigir y controlar debates en línea \\
\hline \multirow{8}{*}{$\begin{array}{l}\text { COMPETENCIAS } \\
\text { SOCIALES }\end{array}$} & Hrastinski et al. (2018) & Fomentar la discusión \\
\hline & $\begin{array}{l}\text { Borges et al. (2014); Tenório et al. } \\
\text { (2016) }\end{array}$ & $\begin{array}{l}\text { Utilizar un lenguaje claro, amigable y } \\
\text { cordial }\end{array}$ \\
\hline & $\begin{array}{l}\text { Hrastinski et al. (2018); Metz y } \\
\text { Bezuidenhout (2018) }\end{array}$ & Fomentar la Reflexión \\
\hline & Hrastinski et al. (2018) & $\begin{array}{llll}\begin{array}{l}\text { Capacidad para } \\
\text { emocional }\end{array} & \text { proporcionar apoyo } \\
\end{array}$ \\
\hline & Adnan (2018) & Capacidad de comunicación \\
\hline & Chien et al. (2016); Adnan (2018) & Consideraciones éticas y morales \\
\hline & $\begin{array}{l}\text { Murphy et al. (2011); Borges et al. } \\
\text { (2014); Tenório et al. (2016); Li } \text { et } \\
\text { al. (2017) }\end{array}$ & Motivar a los estudiantes \\
\hline & $\begin{array}{l}\text { Murphy et al. (2010); Li et al. } \\
\text { (2017); Metz y Bezuidenhout } \\
\text { (2018); Campbell et al. (2019); }\end{array}$ & $\begin{array}{l}\text { Proporcionar un entorno en línea } \\
\text { acogedor, amigable, confiable y } \\
\text { estimulante }\end{array}$ \\
\hline
\end{tabular}

El papel del tutor en la enseñanza de la educación a distancia: una revisión sistemática sobre el enfoque de habilidades. Massuga, Soares y Doliveira. 
RED. Revista de Educación a Distancia. Núm. 66, Vol. 21. Artíc. 9, 30-Abril -2021

DOI: http://dx.doi.org/10.6018/red.435871

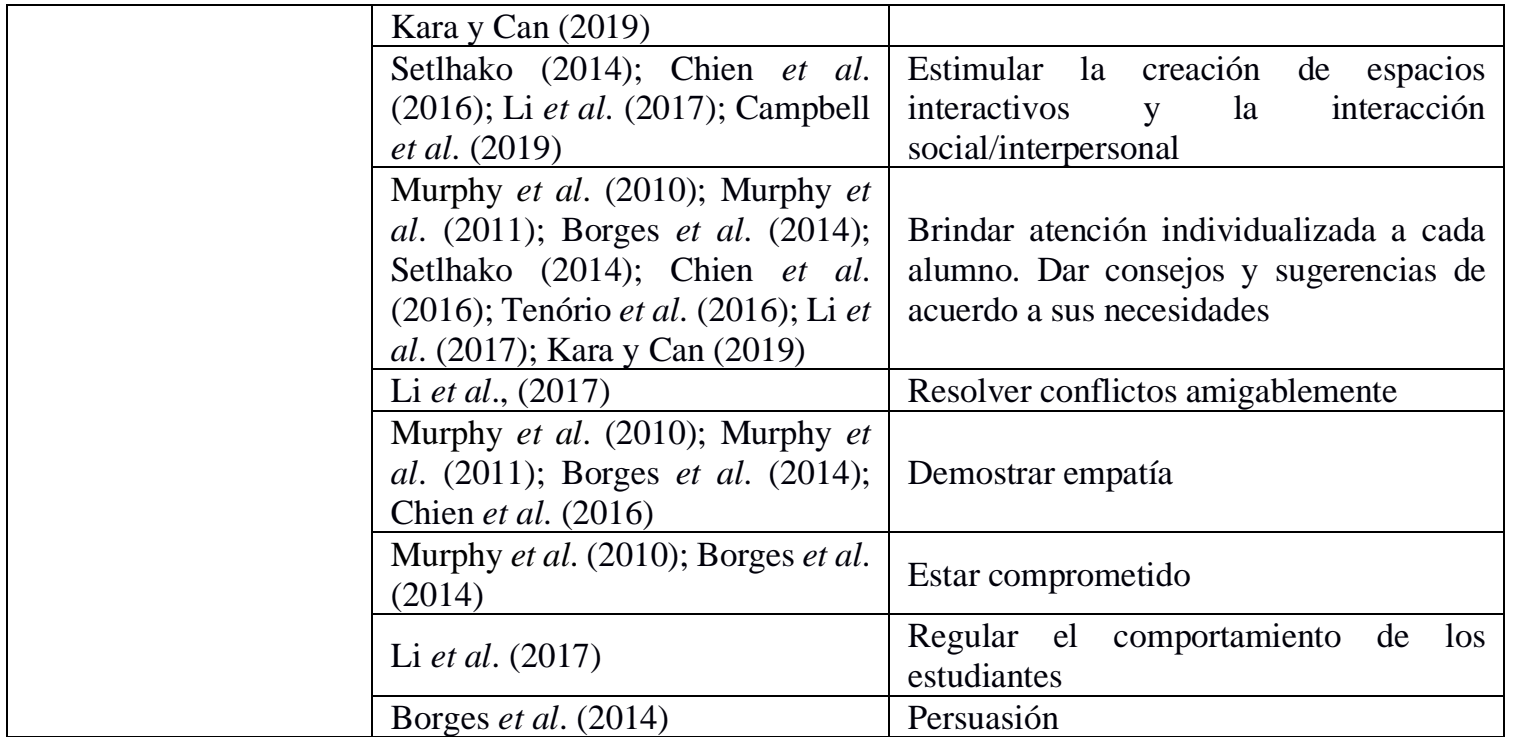

Fuente: Datos de investigación (2020).

Figura 3 - Representación de las competencias del tutor según el número de citas

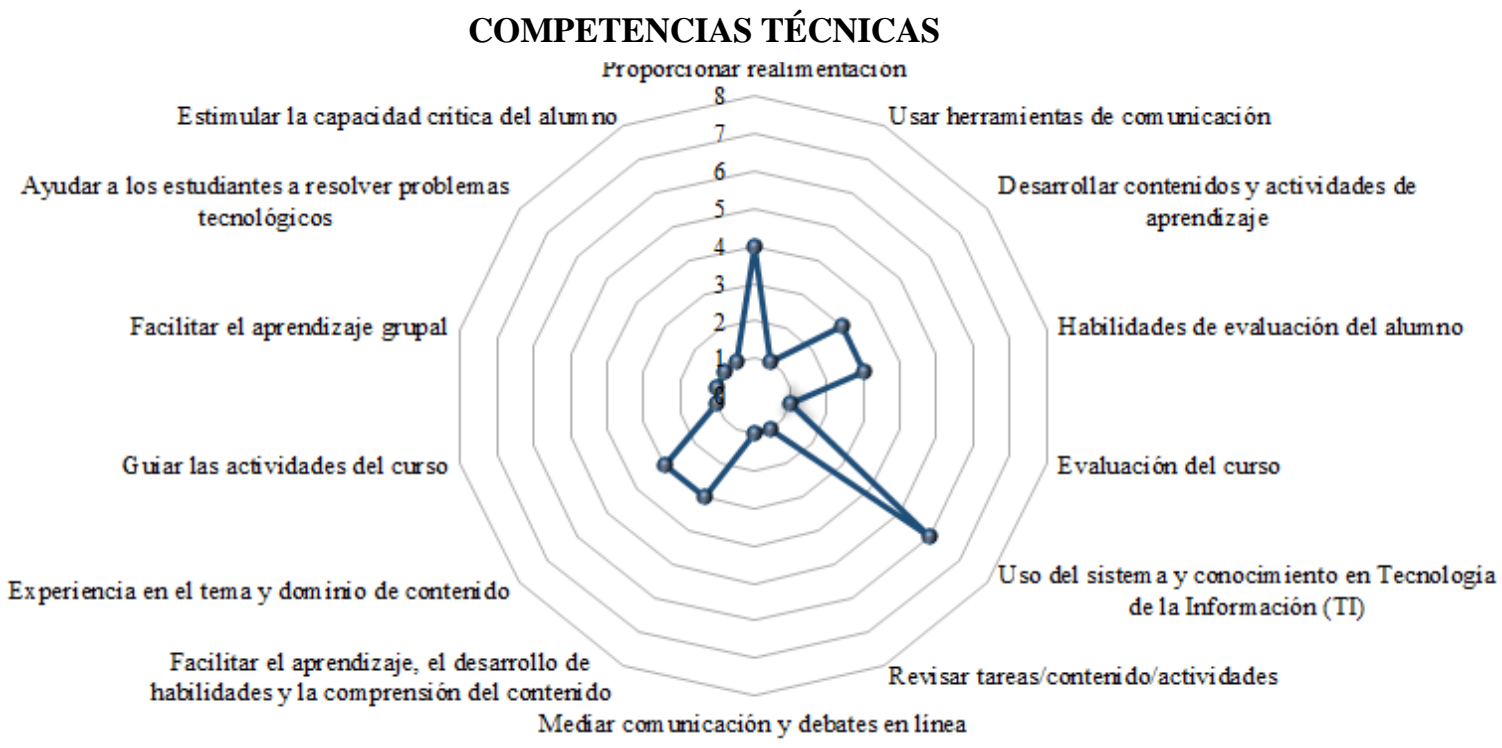

El papel del tutor en la enseñanza de la educación a distancia: una revisión sistemática sobre el enfoque de habilidades. Massuga, Soares y Doliveira. 

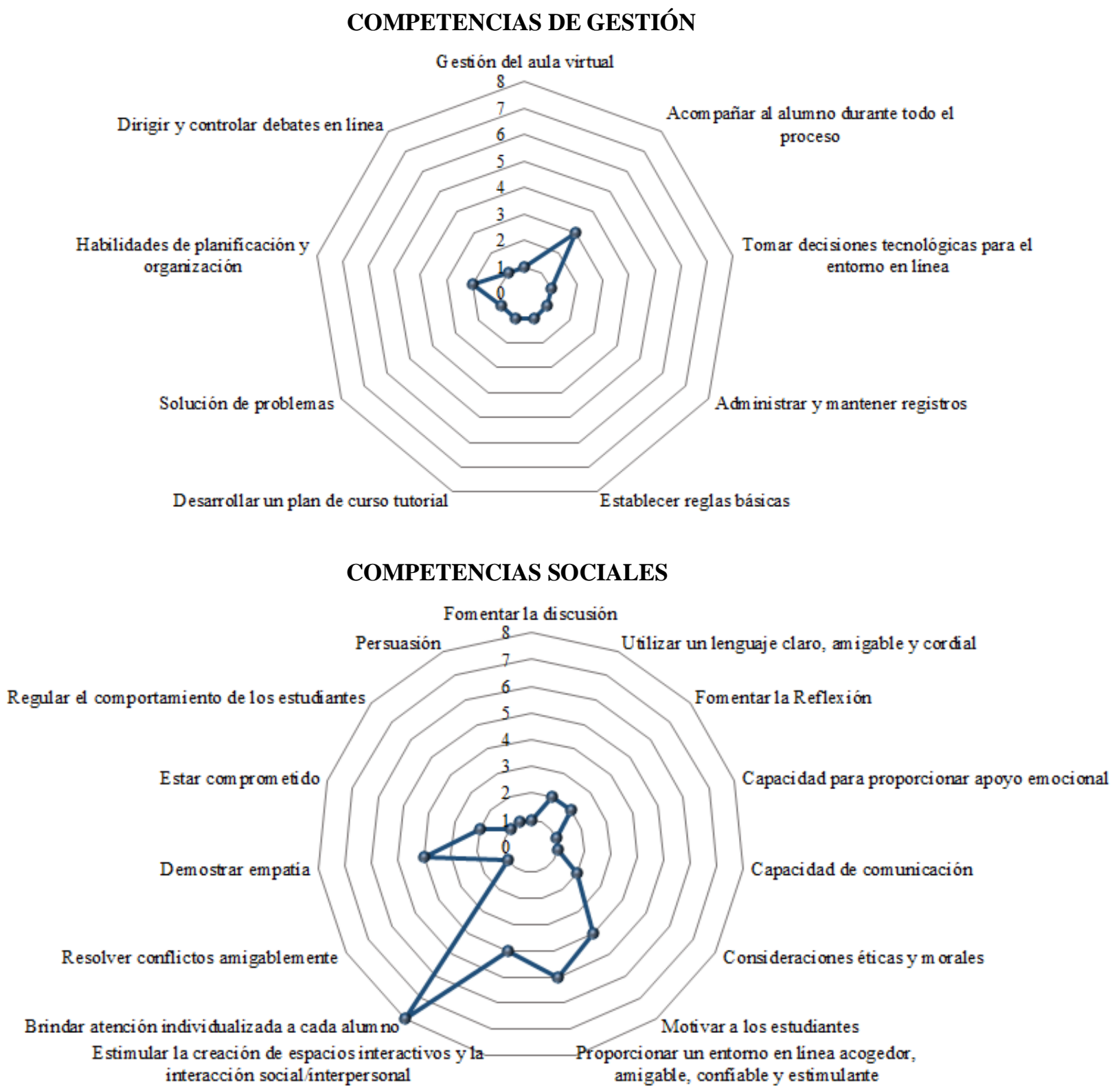

Fuente: Datos de investigación (2020).

Se observa que entre las habilidades más citadas, se encuentra la capacidad de brindar atención individualizada a cada alumno, con 8 menciones, lo que resalta la importancia de considerar las necesidades individuales en el proceso de interacción tutor/alumno. En la secuencia, se encuentra la competencia utilizar el sistema y el conocimiento en tecnología de la información (6 menciones), seguido de proporcionar un entorno en línea acogedor, amigable, confiable y estimulante (5 menciones); demostrar empatía; fomentar la creación de espacios interactivos y la interacción social/interpersonal; motivar a los estudiantes y proporcionar realimentación (4 menciones).

De estas competencias más citadas (7), se observa que la gran mayoría se refiere a competencias sociales (5), lo que confirma las notas de Li et al. (2017), cuando afirman

El papel del tutor en la enseñanza de la educación a distancia: una revisión sistemática sobre el enfoque de habilidades. Massuga, Soares y Doliveira. 
que la prioridad de las competencias en educación a distancia se concentra en una perspectiva constructivista y conectivista.

Sin embargo, a pesar del énfasis en el factor social, aún se evidencian dificultades y desafíos, por ejemplo, en la competencia para proporcionar apoyo emocional (Hrastinski et al., 2018) y en las brechas entre las competencias y los roles que esperan ser cumplido considerando este factor (Adnan, 2018). Por otro lado, los autores también denotan dificultades en relación con las competencias técnicas considerando, por ejemplo, desafíos en relación con el conocimiento tecnológico necesario (Adnan, 2018), a la intervención en el entorno virtual (Borges et al., 2014), a la retroalimentación individualizada a todos los alumnos (Setlhako, 2014) y para evaluar el aprendizaje considerando criterios objetivos y subjetivos (Tenório et al., 2016). Aún así, se evidenciaron dificultades relacionadas con la estimulación de la interacción del estudiante en sistemas sincrónicos (Setlhako, 2014; Campbell et al., 2019). Dada la gran relevancia de estas competencias, confirmada por el número de menciones en relación con ellas, es necesario centrarse en su desarrollo y mejora para ayudar en el desempeño práctico efectivo y eficiente del tutor, contribuyendo también a la calidad de la enseñanza y aprendizaje significativa por parte del alumnado.

En general, se observa en relación a los grupos competenciales divididos según la clasificación propuesta por Grzybowka y Lupicka (2017), que las competencias técnicas asociadas al desempeño de la función profesional corresponden principalmente a los conocimientos tecnológicos para la interacción y las competencias didácticopedagógicas necesarias la ejecución de las actividades del tutor en la educación a distancia. Considerando las competencias gerenciales evidenciadas, se observa su relación con la ejecución de actividades administrativas, burocráticas y organizacionales en las instituciones educativas, involucrando resolución de problemas, planificación, organización, control, toma de decisiones y liderazgo. A su vez, se demostró que las competencias sociales están fundamentalmente vinculadas a las interacciones con los estudiantes para mejorar el aprendizaje. Abarcan principalmente la capacidad de construir un ambiente en línea agradable, con buenas relaciones, además de la capacidad de brindar apoyo y motivación para satisfacer las necesidades individuales que presentan los estudiantes.

Estas consideraciones se resumen en la Figura 04, que representa uma síntesi de los grupos de competencias asociadas al pepel del tutor de educación a distancia.

El papel del tutor en la enseñanza de la educación a distancia: una revisión sistemática sobre el enfoque de habilidades. Massuga, Soares y Doliveira. 
Figura 4 - Síntesis de grupos de competencias asociadas al papel del tutor en la educación a distancia

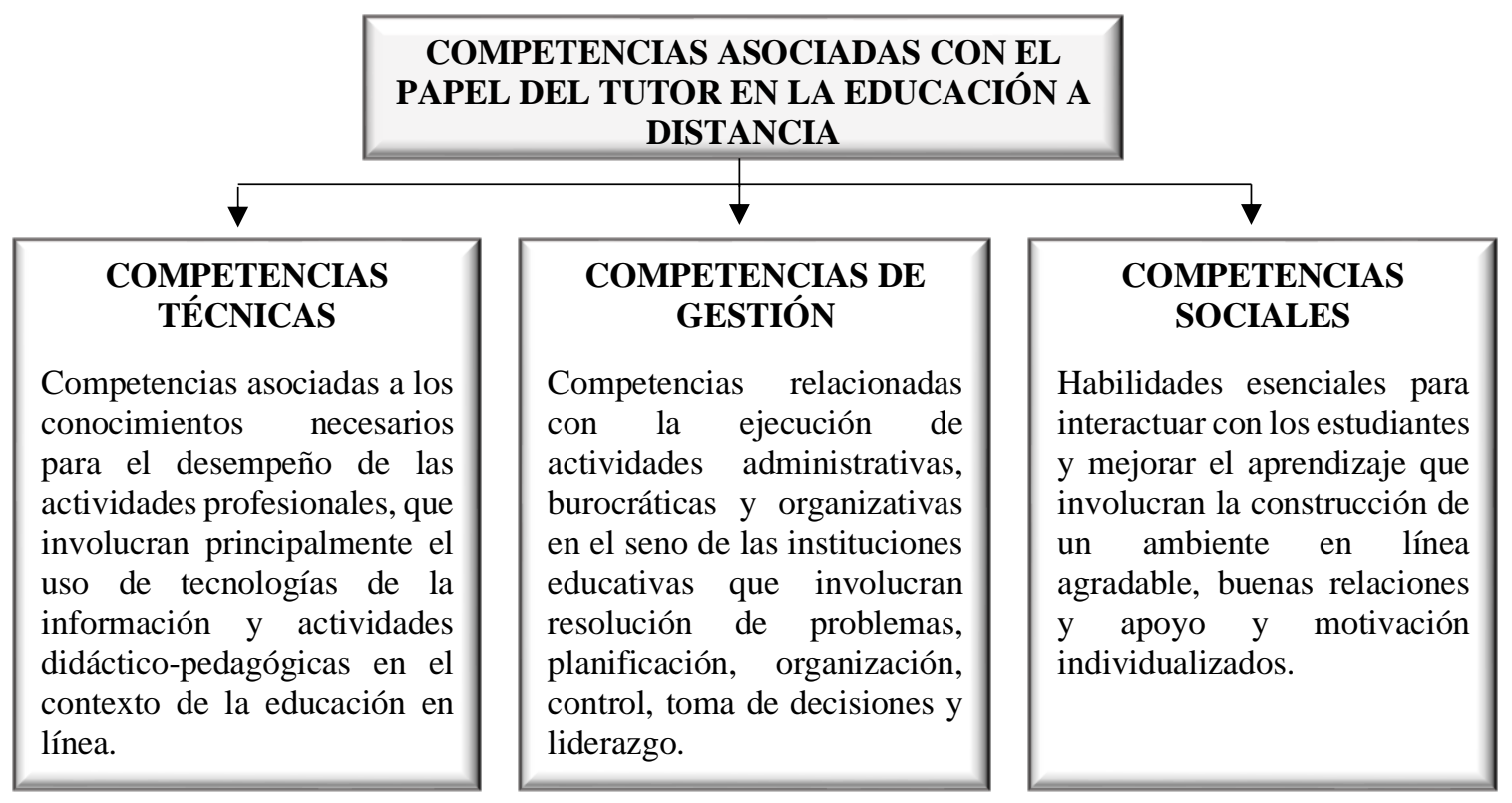

Fuente: Los autores (2020)*.

Los resultados aquí presentados denotan, por tanto, las particularidades de los estudios que describen el enfoque de competencias asociado a la función de tutoría permitiendo la divulgación de las competencias técnicas, gerenciales y sociales vinculadas al tutor en la educación a distancia, los aspectos más destacados y vacíos aún evidenciados, la que responde a los objetivos de esta investigación.

\section{Consideraciones finales}

Este estudio tuvo como objetivo principal identificar las competencias relacionadas con el desempeño del tutor en la modalidad de aprendizaje a distancia. Para esto, se realizó una revisión sistemática, utilizando la metodología Methodi Ordinatio, que resultó en la evaluación de 12 artículos.

El tutor tiene un papel fundamental en la educación a distancia, cuyo desempeño y actividades son diferentes de la modalidad presencial. Es él quien monitorea el proceso de enseñanza-aprendizaje y brinda apoyo al estudiante para enfrentar los desafíos durante el desarrollo del curso. El tutor busca mejorar la interacción y la construcción del conocimiento para lograr el objetivo de aprendizaje. De esta manera, diversos conocimientos, habilidades y actitudes, es decir, un conjunto apropiado de competencias, se hacen necesarios para el desempeño efectivo de sus funciones.

Entre las competencias técnicas, gerenciales y sociales descritas en este estudio, se destacan las siguientes: la capacidad de brindar atención individualizada a los estudiantes, la competencia para utilizar los sistemas y el conocimiento asociados con la tecnología de la información, la competencia para proporcionar un entorno en línea acogedor, amigable, de confianza y estimulante, la competencia para demostrar empatía,

* Clasificación adoptada según lo especificado por Grzybowka y Lupicka (2017).

El papel del tutor en la enseñanza de la educación a distancia: una revisión sistemática sobre el enfoque de habilidades. Massuga, Soares y Doliveira. 
la competencia para fomentar la creación de espacios interactivos y la interacción social/interpersonal, la competencia para motivar a los estudiantes y la competencia proporcionar retroalimentación, que, en gran medida, se dirigen a la perspectiva social.

Aunque importantes, los estudios muestran dificultades aún presentes con respecto a las competencias dirigidas al uso de tecnologías, la provisión de retroalimentación individualizada, la evaluación considerando criterios objetivos y subjetivos y cuestiones sociales relacionadas con la motivación y la dificultad de estimular la participación y la interacción de estudiantes en clases sincrónicas, lo que lleva a la necesidad de una dirección orientada al desarrollo de estas competências para la práctica efectiva del tutor en la asignación de sus funciones.

Esta investigación contribuye con una perspectiva que puede ayudar a los tutores y universidades a mejorar su desempeño relacionado con el desarrollo de competencias clave, esenciales para la calidad de la educación ofrecida. Debido a la literatura aún limitada, reflejada en la selección de solo 12 estudios, y dado el creciente contexto de la educación a distancia, se debe prestar mayor atención a la discusión de sus particularidades, como es el caso de la actuacíon del tutor en esta modalidad de enseñanza. De esta forma, el presente trabajo no agota el tema, posibilitando futuras investigaciones desde diferentes perspectivas en áreas de psicología educativa y pedagogía, por ejemplo. Además, la investigación que los puntos a las tendencias futuras con respecto a la dirección de la educación a distancia en relación con los nuevos medios y tecnologías digitales y el desarrollo de competencias asociadas también puede ser llevado a cabo.

Presentación del manuscrito: 07 de julio de 2020

Fecha de aprobación: 05 de septiembre de 2020

Fecha de publicación: 30 de abril de 2021

Massuga, F., Soares, S., Y Doliveira, S. L. D. (2021). El papel del tutor en la enseñanza de la educación a distancia: una revisión sistemática sobre el enfoque de habilidades. RED. Revista Educación a Distancia, 21(66). http://dx.doi.org/10.6018/red.435871

\section{Financiación}

Esta investigación no ha recibido ninguna subvención específica de los organismos de financiación en los sectores públicos, comerciales o sin fines de lucro.

\section{Referencias}

Adnan, M. (2018). Professional development in the transition to online teaching: the voice of entrant online instructors. ReCALL, $30 \quad$ (1), 88-111. https://doi.org/10.1017/S0958344017000106

Barros, A. J. S., \& Lehfeld, N. A. S. (2007). Fundamentos de metodologia científica. São Paulo: Pearson Prentice Hall.

El papel del tutor en la enseñanza de la educación a distancia: una revisión sistemática sobre el enfoque de habilidades. Massuga, Soares y Doliveira. 
Bezerra, M. A., \& Carvalho, A. B. G. (2011). Tutoria: concepções e práticas na educação a distância. In: R. P. Sousa, F. M. C. S. C. Miota, \& A. B. G. Carvalho. Tecnologias digitais na educação [online]. Campina Grande: EDUEPB (pp. 236-258).

Borges, J. P. F., Coelho Junior, F. A., Faiad, C., \& Rocha, N. F. D. (2014). Diagnóstico de competências individuais de tutores que atuam na modalidade a distância. Educação e Pesquisa, 40 (4), 935-951. http://dx.doi.org/10.1590/s151797022014121642

Brasil. (2017). Decreto $\mathrm{n}^{\circ} 9.057$, de 25 de maio de 2017. Regulamenta o art. 80 da Lei $N^{o}$ 9.394, de 20 de dezembro de 1996, que estabelece as diretrizes e bases da educação nacional. Brasília, DF.

Campbell, A., Gallen, A. M., Jones, M. H., \& Walshe, A. (2019). The perceptions of STEM tutors on the role of tutorials in distance learning. Open Learning: the Journal of Open, Distance and e-Learning, 34 (1), 89-102. https://doi.org/10.1080/02680513.2018.1544488

Chien, C. F., Liao, C. J., Walters, B. G., \& Lee, C. Y. (2016). Measuring the moral reasoning competencies of service-learning e-tutors. Journal of Educational Technology \& Society, $19 \quad$ 269-281. https://www.jstor.org/stable/jeductechsoci.19.3.269

Denyer, D., \& Tranfield, D. (2009). Producing a systematic review. In: D. A. Buchanan, \& A. Bryman. The Sage handbook of organizational research methods. Sage Publications (pp. 671-689).

Dias, I. S. (2010). Competências em educação: conceito e significado pedagógico. Psicologia Escolar e Educacional, $14 \quad$ (1), 73-78. http://dx.doi.org/10.1590/S1413-85572010000100008

Donato, H., \& Donato, M. (2019). Etapas na condução de uma revisão sistemática. Acta Médica Portuguesa, 32 (3), 227-235. 10.20344/amp.11923

Fleury, M. T. L., \& Fleury, A. (2001). Construindo o conceito de competência. Revista de administração contemporânea, 5, 183-196. https://doi.org/10.1590/S141565552001000500010

Gomes, L. F. (2013). EAD no Brasil: perspectivas e desafios. Avaliação: Revista da Avaliação da Educação Superior, 18 (1), 13-22. https://doi.org/10.1590/S141440772013000100002

Grzybowska, K., \& Łupicka, A. (2017). Key competencies for Industry 4.0. Economics \& Management Innovations, $1 \quad$ 250-253. https://doi.org/10.26480/icemi.01.2017.250.253

Hrastinski, S., Cleveland-Innes, M., \& Stenbom, S. (2018). Tutoring online tutors: Using digital badges to encourage the development of online tutoring skills. British journal of educational technology, 49 (1), 127-136. https://doi.org/10.1111/bjet.12525

Instituto Nacional de Estudos e Pesquisas Educacionais [INEP]. (2019). Censo da educação superior 2018: notas estatísticas. Brasília-DF: INEP/MEC. Obtenido de http://download.inep.gov.br/educacao_superior/censo_superior/documentos/2019/ce nso_da_educacao_superior_2018-notas_estatisticas.pdf en 06/06/2020 .

El papel del tutor en la enseñanza de la educación a distancia: una revisión sistemática sobre el enfoque de habilidades. Massuga, Soares y Doliveira. 
Kara, M., \& Can, G. (2019). Master's students' perceptions and expectations of good tutors and advisors in distance education. International Review of Research in Open and Distributed Learning, 20 (2), 1-19. https://doi.org/10.19173/irrodl.v20i2.3674

Kopp, B., Matteucci, M. C., \& Tomasetto, C. (2012). E-tutorial support for collaborative online learning: an explorative study on experienced and inexperienced etutors. Computers $\quad \& \quad$ Education, $58 \quad 12-20$. https://doi.org/10.1016/j.compedu.2011.08.019

Lee, K. (2017). Rethinking the accessibility of online higher education: a historical review. The Internet and Higher Education, 33, 15-23. https://doi.org/10.1016/j.iheduc.2017.01.001

Lee, H. J., Hong, Y., \& Choi, H. (2017). Perceptions of tutoring roles and psychological distance among instructors, tutors and students at a Korean university. Higher Education Research \& Development, 36 (1), 143-157. https://doi.org/10.1080/07294360.2016.1177811

Li, S., Zhang, J., Yu, C., \& Chen, L. (2017). Rethinking distance tutoring in e-learning environments: A study of the priority of roles and competencies of Open University Tutors in China. International Review of Research in Open and Distributed Learning, 18 (2), 189-212. https://doi.org/10.19173/irrodl.v18i2.2752

Marinho-Araujo, C. M., \& Almeida, L. S. (2016). Abordagem de competências, desenvolvimento humano e educação superior. Psicologia: Teoria e Pesquisa, 32 (1), 1-10. https://doi.org/10.1590/0102-3772e32ne212

Marins, E. N., \& Silva, H. D. (2015). Um breve estudo sobre a educação a distância no Brasil e suas vicissitudes. Educação a Distância, Batatais, 5 (2), 67-80.

Martins, O. B. (2003). Teoria e prática tutorial em educação a distância. Educar em Revista, 21, 01-19. https://doi.org/10.1590/0104-4060.288

Metz, N., \& Bezuidenhout, A. (2018). An importance-competence analysis of the roles and competencies of e-tutors at an open distance learning institution. Australasian Journal of Educational Technology, 34 (5), 27-43. https://doi.org/10.14742/ajet.3364

Murphy, L. M, Shelley, M. A., \& Baumann, U. (2010). Qualities of effective tutors in distance language teaching: student perceptions. Innovation in Language Learning and Teaching, 4 (2), 119-136. https://doi.org/10.1080/17501220903414342

Murphy, L. M., Shelley, M. A., White, C. J., \& Baumann, U. (2011). Tutor and student perceptions of what makes an effective distance language teacher. Distance Education, 32 (3), 397-419. https://doi.org/10.1080/01587919.2011.610290

Pagani, R. N., Kovaleski, J. L., \& Resende, L. M. M. de. (2015). Methodi Ordinatio: a proposed methodology to select and rank relevant scientific papers encompassing the impact factor, number of citation, and year of publication. Scientometrics, 105 (3), 2109-2135. https://doi.org/10.1007/s11192-015-1744-X

Pagani, R. N., Kovaleski, J. L., \& Resende, L. M. M. de. (2017). Avanços na composição da Methodi Ordinatio para revisão sistemática de literatura. Ciência da Informação, 46 (2), 161-187.

El papel del tutor en la enseñanza de la educación a distancia: una revisión sistemática sobre el enfoque de habilidades. Massuga, Soares y Doliveira. 
Qayyum, A., \& Zawacki-Richter, O. (2019). The state of open and distance education. In: O. Zawacki-Richter \& A. Qayyum. Open and Distance Education in Asia, Africa and the Middle East. Singapore: Springer (pp. 125-140).

Rezende, E. (2000). O livro das competências. Rio de Janeiro: Qualitymark.

Richardson, R. J. (2010). Pesquisa social: métodos e técnicas. São Paulo: Atlas.

Setlhako, M. A. (2014). Competencies required for the facilitation of online learning courses: an online teaching assistant competency based approach. Mediterranean Journal of Social Sciences, 5 (23), 1405-1405. https://doi.org/10.5901/mjss.2014.v5n23p1405

Silva, S. A., Campos, I. M. S., Melo, M. S. M., \& Rodrigues, J. F. (2014, octubre). Tutoria e o processo de ensino aprendizagem na EAD: um estudo de caso no SENAC-RN. Actas del Congresso Internacional ABED de Educação a Distância. Curitiba, PR, Brasil, 20, p. 1-10.

Skorková, Z. (2016). Competency models in public sector. Procedia-Social and Behavioral Sciences, 230, 226-234. https://doi.org/10.1016/j.sbspro.2016.09.029

Sousa, M. G. T. O, \& Barbosa, M. D. F. N. (2018). A aplicação da gestão por competências nos processos de gestão de pessoas. REUNIR - Revista de Administração Contabilidade e Sustentabilidade, $8 \quad$ (3), 31-46. https://doi.org/10.18696/reunir.v8i3.828

Tenório, A., Teles, S. A., \& Tenório, T. (2016). Levantamento de competências pedagógicas necessárias a tutores da educação a distância. RIED - Revista Iberoamericana de Educación a Distancia, $19 \quad$ (1), 183-207. https://doi.org/10.5944/ried.19.1.13842 